$<$ Short Communication $>$

\title{
Seroprevalence of Coxiella burnetii infection in cattle on Ulleung Island, Korea
}

\author{
Min-Goo Seo ${ }^{1,2}$, In-Ohk Ouh ${ }^{1}$, Young-Hoan Kim ${ }^{3}$, Joong-Kew Kim ${ }^{3}$, Oh-Deog Kwon ${ }^{2}$, Dongmi Kwak ${ }^{2,4, *}$ \\ ${ }^{1}$ Animal and Plant Quarantine Agency, Gimcheon 39660, Korea \\ ${ }^{2}$ College of Veterinary Medicine, Kyungpook National University, Daegu 41566, Korea \\ ${ }^{3}$ Gyeongbuk Veterinary Service Laboratory, Daegu 41405, Korea \\ ${ }^{4}$ Cardiovascular Research Institute, Kyungpook National University, Daegu 41944, Korea
}

(Received: July 4, 2018; Revised: August 4, 2018; Accepted: August 14, 2018)

\begin{abstract}
We assessed the seroprevalence of Coxiella burnetii (C. burnetii) in cattle on Ulleung Island, Korea in a population-based follow up study for 4 years and determined the spatial distribution and risk factors associated with C. burnetii. The seroprevalence of $C$. burnetii was determined to be 1.4-2.0\% during 2011-2014. Overall, nine cattle from three farms that tested seropositive showed $C$. burnetii antibody seroconversions between 2011 and 2014 . The number of seropositive cattle was low, suggesting that movement of and contact between animals was possible risk factors for the transmission of $C$. burnetii.
\end{abstract}

Keywords: Coxiella burnetii, Ulleung Island, animal population groups study, cattle, enzyme-linked immunosorbent assay

Coxiella burnetii (C. burnetii) is the causative agent of $\mathrm{Q}$ fever, a zoonotic coxiellosis [7]. C. burnetii infection in animals causes abortions, premature delivery and weakness in offspring [8]. In cattle, Q fever is normally asymptomatic [7]. However, the abortion rate can reach $5 \%$ in a herd of naïve cattle that contract $C$. burnetii [15]. Various factors have been associated with the transmission of $C$. burnetii in domestic animals, including breeding system, herd density and other unidentified aspects. The main transmission route of infection by $C$. burnetii in humans is ingestion or inhalation of aerosolized pathogens that are spread in the environment by infected animals after abortion or parturition [7]. Bacterial aerosols can be transferred for at least $30 \mathrm{~km}$ by wind [14], and wind plays a role in $C$. burnetii transmission, an epidemiologic factor. Ticks can also transmit Coxiella both transstadially and transovarially to their progenies, acting as a reservoir and possible vector [7].

In Korea, although there have been several investigations on $C$. burnetii in cattle $[3,6,10]$, these were limited to serologic studies of $C$. burnetii antibodies in the Korean mainland. A recent study has revealed serologic and molecular evidence of $C$. burnetii at national breeding stock farms on the mainland of Korea [12], additional periodical tracking data are needed for epidemiological analysis.

Ulleung Island, geographically the second largest island in Korea, is located $130 \mathrm{~km}$ off the east coast of the Korean peninsula. In this island, due to local special industry of Korean traditional cattle, native Korean cattle breeds such as tiger cattle (brindle-pattern in Chikso) were only raised in restricted region of Korea. Therefore, the movement of cattle from the Korean mainland to Ulleung Island has been strictly regulated by the government. Moreover, there is little movement of cattle among farms on Ulleung Island relative to that among farms on the mainland, where cattle movement is frequent and difficult to track. Thus, Ulleung Island is one of the best places for monitoring and surveillance of animal diseases. To protect them from disease, all cattle on Ulleung Island are tested annually for several infectious diseases, including bovine brucellosis, tuberculosis, leucosis and paratuberculosis, as well as neosporosis, by a government-run local veterinary institute since 2007 [11]. However, whether $C$. burnetii is present on Ulleung Island and, if so, the risk factors associated with these infections remain unknown.

The lack of standardized surveillance strategies complicates comparisons of results from previous studies and limits insights into the epidemiologic determinants of transmission of this pathogen [2]. Asymptomatically infected cattle are able to persistently shed $C$. burnetii in milk for several months [1] and long-term secretion of C. burnetii over several months or even years is possible. Therefore, we determined the $C$. burnetii infection status of cattle on Ulleung Island, Korea, a region with special epidemiologic and geographic

*Corresponding author

Tel: +82-53-950-7794, Fax: +82-53-950-5955

E-mail: dmkwak@knu.ac.kr 
conditions using an enzyme-linked immunosorbent assay (ELISA).

This study, conducted between 2011 and 2014, did not receive approval from the Institutional Animal Care and Use Committee at Kyungpook National University, as this committee evaluates laboratory animals maintained in indoor facilities and does not regulate research with outdoor animals. However, under the regulatory "Act on the Prevention of Contagious Animal Disease", national and local veterinary institutes in Korea conducted control measures in accordance with annual infectious animal disease control programs. Blood samples were collected from cattle by practicing veterinarians at the local government-run veterinary institutes during monitoring, surveillance and treatment, or during regular check-ups after the receipt of verbal consent from the cattle owners. This blood collection at the government-run veterinary institutes was carried out according to the administrative rules of the Ministry of Agriculture, Food and Rural Affairs, Korea.

The total number of cattle reared in Korea including mainland and islands, was recorded as 3,087,767 in 2015, of which 402 were raised on Ulleung Island based on the data of year 2015 [5]. All cattle from every farm reared on Ulleung Island were examined in the study each year between 2011 and 2014. The island has a mild oceanic climate with an area of about $72.9 \mathrm{~km}^{2}$, with a mean annual precipitation of $1,383.4 \mathrm{~mm}$ and mean annual temperature of $12.4^{\circ} \mathrm{C}$ [9]. Ulleung Island is divided into three administrative regions: Ulleung-eup, Seo-myeon and Buk-myeon (Fig. 1). In this study, blood samples were collected from 760 cattle from 54 farms, 597 cattle from 51 farms, 575 cattle from 49 farms and 625 cattle from 49 farms, each year from 2011 to 2014, respectively. Data on the region, age, sex and breed of cattle sampled were recorded.

Antibodies against $C$. burnetii were detected in cattle sera using an ID Screen Q Fever Indirect Multi-species ELISA kit (IDvet, France), which was carried out following the manufacturer's instructions using Phase I and II purified antigens of $C$. burnetii. Seroconversion was defined as the presence of antibodies against $C$. burnetii in the second but not the first sample collected between 2011 and 2014, suggesting a new infection in the interval between when the first and second sample were obtained.

The Chi-square test was used to determine significant differences between multiple groups, with $p$ values $<0.05$ regarded as statistically significant. GraphPad Prism (ver. 5.04; GraphPad Software, USA) was used for the statistical analyses.

As shown in Table 1, the seropositivity of $C$. burnetii determined by ELISA (1.4-2.0\%) was low between 2011 and 2014. During the study period, C. burnetii was found on only three farms in the Seo-myeon region. $C$. burnetii seroprevalence on three farms with $C$. burnetii-positive cattle was 5.6\% (3/ $54), 5.9 \%(3 / 51), 6.1 \%(3 / 49)$ and $6.1 \%(3 / 49)$ from 2011 to 2014 , respectively (data not shown). The Seo-myeon region showed the greatest densities of cattle at both the farm and individual levels compared to those of other regions. Moreover, the three farms with cattle testing positive for $C$. burnetii neighbor one another (Fig. 1). By breed, the $C$. burnetii seroprevalence was found to be significantly higher in tiger cattle than in any other breed in 2011 and 2012 ( $p<$

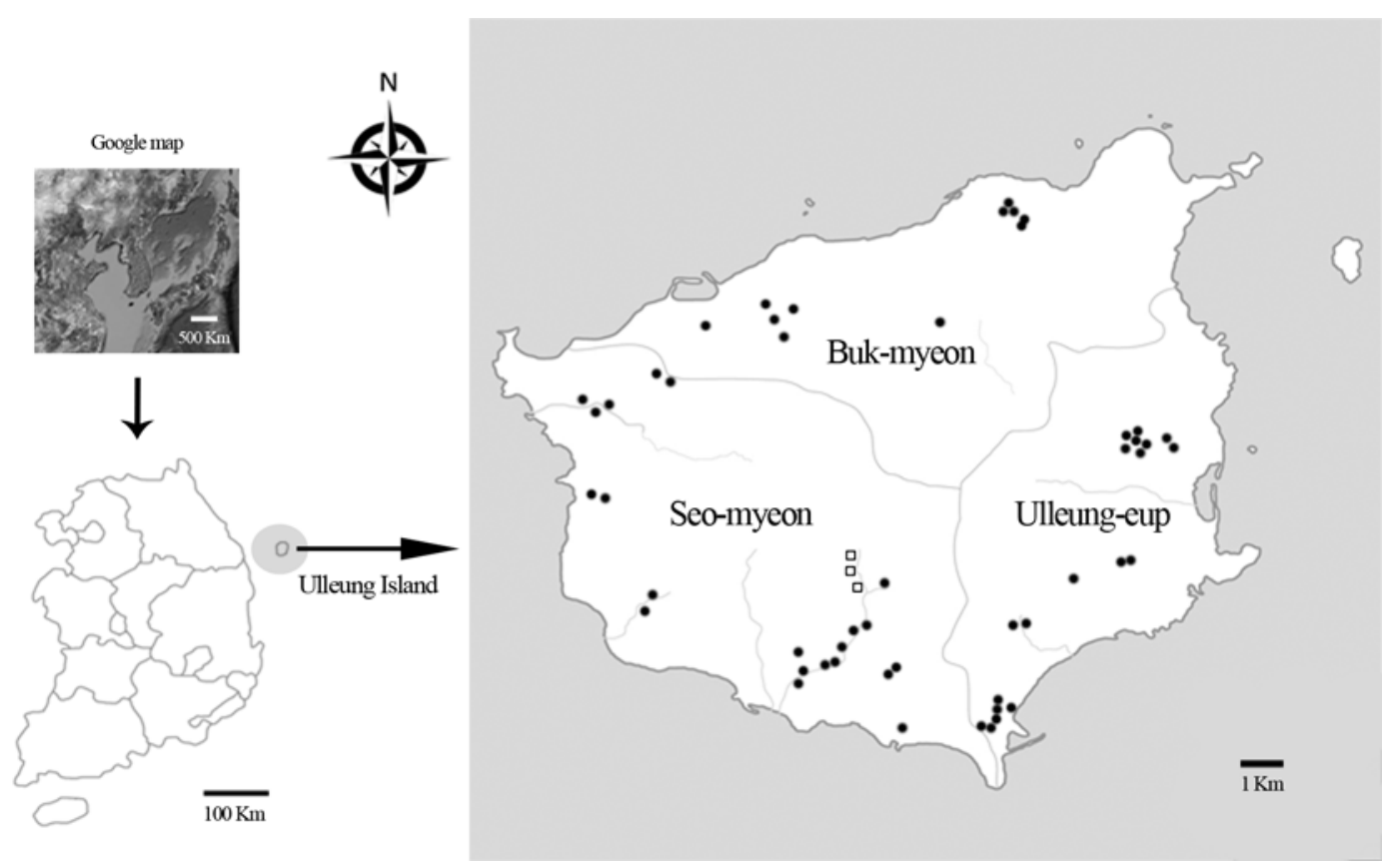

Fig. 1. Spatial distribution of all cattle farms on Ulleung Island, Korea. The three study regions (Ulleung-eup, Seo-myeon and Bukmyeon) are indicated. Dots and squares denote cattle farms found negative and positive for Coxiella burnetii, respectively, between 2011 and 2014. 
Table 1. Seroprevalence of Coxiella burnetii in cattle reared on Ulleung Island according to breed, sex and age of cattle between 2011 and 2014

\begin{tabular}{clcccc}
\hline \hline \multirow{2}{*}{ Group } & Sub-group & \multicolumn{3}{c}{ Number of positive cattle/Number of tested (\%) cattle } \\
\cline { 3 - 6 } & & 2011 & 2012 & 2013 & 2014 \\
\hline \multirow{2}{*}{ Breed } & Brown cattle & $2 / 330(0.6)$ & $0 / 250$ & $2 / 230(0.9)$ & $3 / 323(0.9)$ \\
& Tiger cattle & $13 / 430(3.0)$ & $10 / 347(2.9)$ & $7 / 345(2.0)$ & $6 / 302(2.0)$ \\
& $p$ value & 0.018 & 0.007 & 0.273 & 0.267 \\
Sex & Female & $13 / 431(3.0)$ & $8 / 336(2.4)$ & $8 / 326(2.5)$ & $8 / 370(2.2)$ \\
& Male & $2 / 329(0.6)$ & $2 / 261(0.8)$ & $1 / 249(0.4)$ & $1 / 255(0.4)$ \\
& $p$ value & 0.018 & 0.1273 & 0.0495 & 0.0679 \\
Age & $<2$ & $6 / 448(1.3)$ & $2 / 305(0.7)$ & $1 / 237(0.4)$ & $2 / 336(0.6)$ \\
& $2-3$ & $4 / 257(1.6)$ & $1 / 256(0.4)$ & $2 / 287(0.7)$ & $1 / 235(0.4)$ \\
& $>3$ & $5 / 55(9.1)$ & $7 / 36(19.4)$ & $6 / 51(11.8)$ & $6 / 54(11.1)$ \\
Total & $p$ value & 0.004 & $<0.0001$ & $<0.0001$ & $<0.0001$ \\
\hline
\end{tabular}

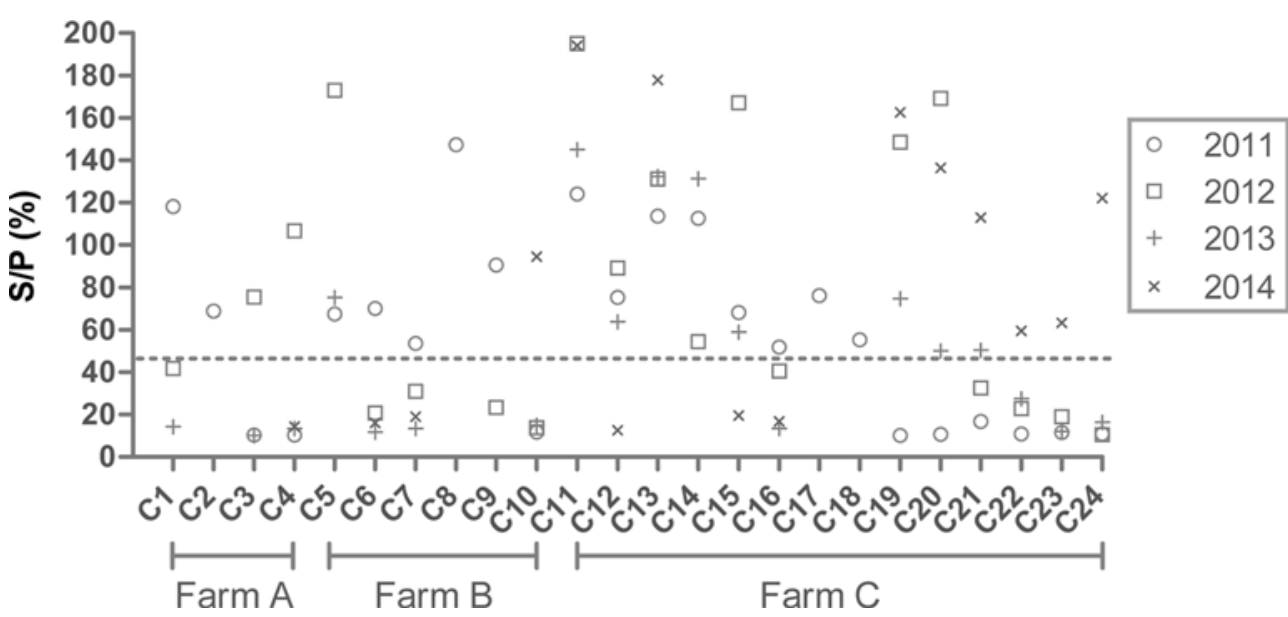

Fig. 2. Comparison of Coxiella burnetii antibody levels in 24 antibody-positive cattle from three farms on Ulleung Island by ELISA between 2011 and 2014. Farm A each had 2 cattle seropositive for C. burnetii antibody in 2011 and 2012; farm B had 5, 1, 1 and 1 cattle seropositive for C. burnetii antibody in 2011, 2012, 2013 and 2014, respectively; and farm C had 8, 7, 8 and 8 cattle seropositive for C. burnetii antibody in 2011, 2012, 2013 and 2014, respectively. Samples yielding an S/P (sample-to-positive control ratio) greater than $50 \%$, which was the cut-off value (horizontal dashed line), were interpreted as positive. C, cattle.

0.05). The prevalence of $C$. burnetii was significantly higher in female cattle than in male cattle in 2011 and $2013(p<$ $0.05)$. The positivity of $C$. burnetii significantly increased with age of the cattle between 2011 and $2014(p<0.05)$.

To identify seroconversions in individual cattle which include all 24 positive cattle during 2011 and 2014, C. burnetii antibody levels in the paired sera of 24 positive cattle (temperately numbered 1 to 24 in this study) on the three farms were compared (Fig. 2). Cattle 3 and 4 on farm A and Cattle 19 and 20 on farm $C$ in 2012, Cattle 21 on farm $C$ in 2013 and Cattle 10 on farm B and Cattle 22-24 on farm C in 2014 showed $C$. burnetii seroconversions. The $C$. burnetii seroprevalence decreased over time on farms A and B, but, on farm $\mathrm{C}$, multiple cattle showed continuous seroconversions between 2011 and 2014 (Fig. 2).
We performed a population-based study with 4 years of follow up on the prevalence of $C$. burnetii in cattle reared on Ulleung Island, Korea. These cattle showed a low seroprevalence of $C$. burnetii between 2011 and 2014. The seroprevalence of $C$. burnetii at the herd and individual levels decreased over time. The low seropositivity found in this study are similar to that described in a previous report of $C$. burnetii seropositivity in cattle of Korea determined by ELISA $(1.3 \% ; 13 / 1,000)$ [3] and by immunofluorescence assay and ELISA $(4.2 \% ; 180 / 4,311)$ [6]. Ulleung Island is a small area and cattle breeding is limited. Additionally, cattle on this island are allowed to graze and roam freely in near grassland. The three positive farms were within $1-2 \mathrm{~km}$ of each other, indicating that the C. burnetii-positive cattle might have been epidemiologically and geographically linked. 
Moreover, these farms are located in the mountain area which has more opportunity to contact vector ticks. Although an examination of human infections on the farms with positive cattle on Ulleung Island was beyond the scope of our study, such an analysis may help elucidate the epidemiology of Q fever in humans and animals. A previous study showed that the presence of antibodies to C. burnetii was significantly associated with specific risk factors in cattle farmers and farm residents, including milking cattle, providing general healthcare to cattle, providing birth assistance to cattle, contacting stillborn calves, urbanization and presence of mice and/or rats in stables [13].

To analyze cattle at the herd level within farms, C. burnetii serum antibody levels in cattle were compared among the three farms. The $C$. burnetii antibody titer varied among the three farms (data not shown). Since farm A and B were good at managing the farms by individual owners, the seroprevalences of $C$. burnetii in these two farms were gradually decreased. The farm $\mathrm{C}$, meanwhile, showed evidence of continuous $C$. burnetii transmission since it was less wellmanaged by a local society. Interestingly, farm $\mathrm{C}$ had the highest number of breeding cattle on Ulleung Island and imported cattle from the mainland more frequently than any other farms on Ulleung Island. Thus, cattle in farm $\mathrm{C}$ had more opportunities for exposure to disease agents than other farms whose cattle do not nearly move to each other due to feature of isolated island region. Therefore, movement of and contact between animals are likely important risk factors for C. burnetii transmission.

In Korea, there are several breeds of cattle, including tiger cattle and brown cattle (Hanwoo) [12]. Tiger cattle showed a higher $C$. burnetii prevalence than any other breed. Tiger cattle are only raised in a restricted region of Ulleung Island and Jeonbuk province of mainland, Korea. In another study on $C$. burnetii, tiger cattle were also more prevalent than brown cattle in mainland of Korea by using both ELISA and PCR [12]. Although it is not clarified yet, these differences in breed may influence the infection rate. C. burnetii seropositivity was higher in female cattle and increased with age. These results were similar to those of a previous study that tested for $C$. burnetii antibody in dairy cattle and showed higher $C$. burnetii positivity in females and an increase in prevalence with age in Gyeongbuk province of Korea [10], a ELISA- and PCR-based study on native Korean goats that showed an increase in positivity with age in the mainland of Korea [4] and a study on cattle on national breeding stock farms that showed higher $C$. burnetii seropositivity in females and an increase in seropositivity with age in the mainland and Jeju Island of Korea [12]. This might be attributed to the high numbers of bacteria shed during parturition [2], which would likely increase exposure of both the females and the herd with the high breeding numbers especially in farm C.

Additionally, seroconversions occurring between 2011 and 2014 were assessed. Overall, nine cattle on the three farms with $C$. burnetii-positive cattle showed seroconversions between
2011 and 2014. The results from farms A and B showed that the seroprevalence of $C$. burnetii in herds decreased over time. Interestingly, on farm $\mathrm{C}$, several cattle seroconverted every year between 2012 and 2014. The data also indicated that $C$. burnetii antibodies could persist for long time in a herd, as two cattle (Cattle 11 and 13) remained seropositive from 2011 to 2014. In Korea, Q fever is classified into the group of contagious animal diseases Type II and culling is recommended to prevent the spread of the disease. Thus, the local authorities should ban the transport of animals in these affected farms and establish an adequate control program.

Cattle movement from the mainland to Ulleung Island has been restricted by the government, resulting in a low risk of C. burnetii infection for cattle on Ulleung Island. In addition, there is less movement of cattle among farms on this island compared to that on the mainland. Probably because of these epidemiologic (animal movement) and geographic (island) restrictions, the rate of $C$. burnetii positivity in cattle was low on Ulleung Island. This may indicate that a quarantine system is important for the prevention of $C$. burnetii infection in both animals and farmers.

Concluding, the results of this 4-year population-based follow-up study on Ulleung Island indicated that $C$. burnetii was only present on three farms, with $C$. burnetii seropositivity of 1.4-2.0\% between 2011 and 2014. This low seroprevalence of $C$. burnetii may have been maintained as the result of well-organized control measures on Ulleung Island. Because the three farms with $C$. burnetii-positive cattle are within 1-2 $\mathrm{km}$ of each other, a quick intervention by the government could lead to the decrease incidence of this disease from Ulleung Island. To ensure that Ulleung Island is free of the causative agent of Q fever, domestic and wild animals should be included in the monitoring list of annual infectious diseases by the local authorities.

\section{References}

1. Guatteo R, Beaudeau F, Joly A, Seegers H. Coxiella burnetii shedding by dairy cows. Vet Res 2007, 38, 849-860.

2. Guatteo R, Seegers H, Taurel AF, Joly A, Beaudeau F. Prevalence of Coxiella burnetii infection in domestic ruminants: a critical review. Vet Microbiol 2011, 149, 1-16.

3. Jang Y, Kim H, Heo I, Park Y, Kim S, Lee M, Choe N. Seroprevalence of Coxiella burnetii in cattle and farm-raised deer in Korea. Afr J Microbiol Res 2011, 5, 4234-4236.

4. Jung BY, Seo MG, Lee SH, Byun JW, Oem JK, Kwak D. Molecular and serologic detection of Coxiella burnetii in native Korean goats (Capra hircus coreanae). Vet Microbiol 2014, 173, 152-155.

5. Korean Statistical Information Service. Number of farms in households and animals in heads by type. Statistics Korea, Daejeon, 2015.

6. Lyoo KS, Kim D, Jang HG, Lee SJ, Park MY, Hahn TW. Prevalence of antibodies against Coxiella burnetii in Korean native cattle, dairy cattle, and dogs in South Korea. Vector Borne Zoonotic Dis 2017, 17, 213-216.

7. Maurin M, Raoult D. Q fever. Clin Microbiol Rev 1999, 
12, 518-553.

8. Muskens J, van Engelen E, van Maanen C, Bartels C, Lam TJGM. Prevalence of Coxiella burnetii infection in Dutch dairy herds based on testing bulk tank milk and individual samples by PCR and ELISA. Vet Rec 2011, 168, 79.

9. National Climate Data Service System. Climate data. Climatological normal. Korea Meteorological Administration, Seoul, 2011.

10. Ouh IO, Seo MG, Do JC, Kim IK, Cho MH, Kwak DM. [Seroprevalence of Coxiella burnetii in bulk-tank milk and dairy cattle in Gyeongbuk province, Korea]. Korean J Vet Serv 2013, 36, 243-248. Korean.

11. Seo MG, Do JC, Ouh IO, Cho MH, Kim JK, Kim YH, Park NC, Kwak DM. [Prevalence of infectious agents in cattle reared in Ulleung island]. Korean J Vet Serv 2011, 34, 303-311. Korean.
12. Seo MG, Ouh IO, Lee SH, Kim JW, Rhee MH, Kwon OD, Kim TH, Kwak D. Prevalence of Coxiella burnetii in cattle at South Korean national breeding stock farms. PLoS One 2017, 12, e0177478.

13. Sun WW, Cong W, Li MH, Wang CF, Shan XF, Qian AD. Coxiella burnetii seroprevalence and risk factors in cattle farmers and farm residents in three northeastern provinces and Inner Mongolia Autonomous Region, China. Biomed Res Int 2016, 2016, 7059196.

14. Tissot-Dupont H, Amadei MA, Nezri M, Raoult D. Wind in November, Q fever in December. Emerg Infect Dis 2004, 10, 1264-1269.

15. Van den Brom R, Vellema P. Q fever outbreaks in small ruminants and people in the Netherlands. Small Rumin Res 2009, 86, 74-79. 